\title{
Effect of anthracycline-based postoperative chemotherapy on blood glucose and lipid profiles in patients with invasive breast cancer
}

\author{
Aiying Qi, Yanping Li, Susu Yan, Huiying Sun, Yuhui Chen \\ Department of General Surgery, the First Medical Center of the Chinese People's Liberation Army (PLA) General Hospital, Beijing, China \\ Contributions: (I) Conception and design: A Qi, Y Chen; (II) Administrative support: A Qi, Y Chen; (III) Provision of study materials or patients: All \\ authors ; (IV) Collection and assembly of data: All authors; (V) Data analysis and interpretation: All authors; (VI) Manuscript writing: All authors; (VII) \\ Final approval of manuscript: All authors. \\ Correspondence to: Yuhui Chen. Attending Physician, Department of General Surgery, the First Medical Center of the Chinese PLA General Hospital, \\ 28 Fuxing Road, Haidian District, Beijing, China. Email: cyhnju@sina.com.
}

Backgroundk Recent studies have shown that chemotherapy can cause abnormal glucose and lipid metabolism in breast cancer patients; however, the effects of different chemotherapy regimens on the glucose and lipid profiles in this population remain unclear.

Methods: The clinical data of 141 invasive breast cancer patients who were treated in our center from January 2019 to December 2020 were retrospectively collected. All patients received surgical treatment and postoperative chemotherapy in our center. According to the postoperative chemotherapy regimens, these patients were divided into an observation group ( $\mathrm{n}=100$, treated with anthracycline-based regimens) and a control group ( $n=41$, treated with non-anthracycline-based regimens). Blood glucose and lipid profiles were compared between the 2 groups.

Results: Compared with the control group, the observation group had a significantly higher radiotherapy rate $(74.00 \%$ vs. $43.90 \%, \mathrm{P}=0.001)$ and a significantly higher proportion of patients receiving $>6$ cycles of chemotherapy $(85.00 \%$ vs. $4.88 \%, \mathrm{P}=0.000)$. There were no significant significances in the levels of total cholesterol (TC), triglyceride (TG), low-density lipoprotein (LDL) before and after treatment $(\mathrm{P}>0.05)$ in both groups. TC and high-density lipoprotein (HDL) were not significantly different between the observation group and control group before chemotherapy $(\mathrm{P}>0.05)$. After chemotherapy, fasting blood glucose significantly increased in the observation group $(5.31 \pm 0.98$ vs. $4.96 \pm 0.53, \mathrm{P}=0.031)$, while HDL significantly decreased $(1.08 \pm 0.28$ vs. $1.19 \pm 0.31, \mathrm{P}=0.042)$. Multivariate logistic regression analysis showed that anthracycline-based chemotherapy was a protective factor for increased fasting blood glucose after chemotherapy in invasive cancer breast patients $[\mathrm{P}=0.022$, odds ratio $(\mathrm{OR})=0.227,95 \%$ confidence interval (CI): 0.064-0.808], whereas receiving $>6$ cycles of chemotherapy was a risk factor for increased fasting blood glucose $(\mathrm{P}=0.014$, OR $=4.216,95 \% \mathrm{CI}: 1.337-13.296)$.

Conclusions: Anthracyclines have little effect on fasting blood glucose in breast cancer patients; however, the incidence of abnormal blood glucose metabolism is gradually increasing after prolonged anthracycline use. Compared with other chemotherapy drugs, anthracycline-based chemotherapy has no significant impact on blood lipid metabolism.

Keywords: Anthracyclines; postoperative chemotherapy; invasive breast cancer; blood glucose; blood lipids

Submitted Jan 11, 2021. Accepted for publication May 21, 2021.

doi: $10.21037 / \mathrm{apm}-21-533$

View this article at: http://dx.doi.org/10.21037/apm-21-533 


\section{Introduction}

Breast cancer is the most common malignancy in women. Due to changes in the environment and workplace stress in recent years, there is an upward trend in the incidence of breast cancer, especially among young adults in many areas (1-3). With the increased awareness of this malignancy, breast cancer can be detected and treated early, and in many cases there is a chance for radical resection (4-7). However, breast cancer with micrometastases at diagnosis is difficult to completely remove. Therefore, postoperative adjuvant chemotherapy is often required in some breast cancer patients to substantially reduce the postoperative recurrence rate (8-10). Although chemotherapy can reduce the recurrence rate after surgery, it can also lead to many complications such as bone marrow suppression, hair loss, and gastrointestinal symptoms. Some recent studies have explored the effect of chemotherapy on metabolism. Previous studies had confirmed that the characteristics of blood lipid metabolism were different between invasive breast cancer patients and benign breast tumor patients. Moreover, patients with different molecular types could have different characteristics of blood lipid metabolism. In addition, it has been found that postoperative chemotherapy can cause abnormal blood glucose and lipid metabolism in breast cancer patients (11). However, few studies have investigated the effects of different chemotherapy regimens on blood glucose and blood lipids. There are currently 2 main chemotherapy protocols: anthracycline-based chemotherapy regimens and nonanthracycline-based chemotherapy regimens. Here, we explored the effect of postoperative anthracycline-based chemotherapy regimen on blood glucose and lipids in patients with invasive breast cancer.

We present the following article in accordance with the STROBE reporting checklist (available at http://dx.doi. org/10.21037/apm-21-533).

\section{Methods}

\section{General data}

The clinical data of 141 invasive breast cancer patients who were treated in our center from January 2019 to December 2020 were retrospectively collected. The inclusion criteria were as follows: (I) female breast cancer patients undergoing surgery and chemotherapy in our hospital; (II) aged 18-75 years; (III) having received at least 4 cycles of chemotherapy; and (IV) with complete clinicopathologic data. The exclusion criteria included the following: (I) not undergoing standardized chemotherapy as required; (II) with cardiac, cerebral, pulmonary, and/or other vital organ dysfunction; (III) in the advanced stage, with distant metastasis, and undergoing salvage chemotherapy; (IV) with metastatic or recurrent breast cancer; (V) with abnormal thyroid function; (VI) with accompanying malignant tumors (e.g., gastric cancer); (VII) pregnant or lactating women; and (VIII) with special types of invasive breast cancer. All procedures performed in this study involving human participants were in accordance with the Declaration of Helsinki (as revised in 2013). This study was approved by the ethics committee of the First Medical Center of the Chinese People's Liberation Army General Hospital (2021-166) and individual consent for this retrospective analysis was waived.

\section{Grouping}

According to the postoperative chemotherapy regimens, these patients were divided into an observation group ( $\mathrm{n}=100$, treated with anthracycline-based regimens) and a control group ( $n=41$, treated with non-anthracycline-based regimens).

\section{Treatments}

Anthracycline-based chemotherapy regimen or nonanthracycline-based chemotherapy regimen was given after surgery. In the observation group, the regimens given included AC-T (adriamycin + cyclophosphamide, followed by treatment with Taxol; $n=17)$, EC-TH $(n=14)$ AT $(n=2)$, EC-T (n=56), EC-TPH (n=1), EC (n=4), EC-TH+P (n=2), EC-TP+PD1 $(n=1)$, ET $(n=1)$, ET-C $(n=1)$, and TCE $(n=1)$. In the control group, the regimens given included NP $(n=1)$, TC $(n=21), T C+H(n=4), T C b H(n=1), T C H$ $(n=9), T C H+P(n=2), T H(n=2)$, and TX $(n=1)$. Patients in both groups were given 4 or more cycles of chemotherapy according to the standardized protocols.

\section{Main measures}

The main indicators included the following: (I) fasting blood glucose; and (II) blood lipids, including total cholesterol, triglyceride, high-density lipoprotein (HDL), and low-density lipoprotein (LDL). Other indicators included age, body mass index, involved site(s), history of diabetes, history of hypertension, and size of primary mass; estrogen receptor (ER), progesterone receptor (PR), Ki67, human epidermal growth factor receptor 2 (HER2), 
and lymph node metastasis status; surgical procedure type; occurrence of axillary lymph node dissection, postoperative endocrine therapy, and radiotherapy; and chemotherapy regimen and total chemotherapy cycles.

\section{Definitions}

The positive cutoffs for ER, PR, and Ki-67(\%) were $\geq 10 \%$, $\geq 10 \%$, and $\geq 14 \%$, respectively.

\section{Quality control}

In our study, we designed strict inclusion and exclusion criteria. In addition, we chose objective indicators to avoid recall bias. Finally, we chose two persons to input data independently. If the data was inconsistent, a third person would check it.

\section{Statistical analysis}

Statistical analysis was performed in the SPSS 22.0 software package (IBM Corp., Armonk NY, USA) and a $\mathrm{P}$ value of less than 0.05 was considered statistically significant. Changes in glucose and lipids before and after chemotherapy were analyzed using paired samples $t$-test, and intergroup comparison was performed using independent samples $t$-test. Data are presented as mean \pm standard deviation (SD).

\section{Results}

\section{General data of the two groups}

The general data including age, body mass index, involved site, history of hypertension, history of diabetes, ERpositive rate, PR-positive rate, Ki-67-positive rate, HER2positive rate, surgical procedure type, endocrine therapy rate, and targeted drug therapy rate showed no statistically significant differences between these 2 groups (all $\mathrm{P}>0.05$ ). Compared with the control group, the observation group had a significantly higher radiotherapy rate $(74.00 \%$ vs. $43.90 \%, P=0.001)$ and a significantly higher proportion of patients receiving $>6$ cycles of chemotherapy $(85.00 \% v s$. $4.88 \%, \mathrm{P}=0.000$; Table 1).

\section{Comparisons of glucose and lipid profiles before and after chemotherapy between the two groups}

There were no significant significances in the levels of total cholesterol (TC), triglyceride (TG), LDL before and after treatment $(\mathrm{P}>0.05)$ in both groups. TC and HDL were not significantly different between the observation group and control group before chemotherapy $(\mathrm{P}>0.05)$. After chemotherapy, fasting blood glucose significantly increased in the observation group $(5.31 \pm 0.98$ vs. $4.96 \pm 0.53, \mathrm{P}=0.031)$, while HDL significantly decreased $(1.08 \pm 0.28$ vs. $1.19 \pm 0.31$, $\mathrm{P}=0.042$; Table 2).

\section{Risk factors for increased fasting glucose after chemotherapy in patients with invasive breast cancer}

An anthracycline-based chemotherapy regimen was a protective factor for increased fasting glucose after chemotherapy in patients with invasive breast cancer $[\mathrm{P}=0.022$, odds ratio $(\mathrm{OR})=0.227,95 \%$ confidence interval (CI): 0.064-0.808], whereas receiving $>6$ cycles of chemotherapy was a risk factor for increased fasting blood glucose $(\mathrm{P}=0.014$, OR $=4.216,95 \%$ CI: $1.337-13.296$; Table 3).

\section{Risk factors for reduction of HDL in invasive breast cancer patients after chemotherapy}

Multivariate logistic regression analysis showed that anthracycline-based chemotherapy was not a risk factor for decreased HDL after chemotherapy in invasive cancer breast patients $(\mathrm{P}<0.05$; Table 4$)$.

\section{Discussion}

Anthracyclines are commonly used in the clinical setting. They are the main components of first-line chemotherapy regimens for breast cancer $(12,13)$ and play important roles in the treatment of advanced gastric cancer and breast cancer. Anthracyclines mainly include doxorubicin, epirubicin, piribicin, zoloftomycin, arabicin, idarubicin, and anirubicin $(14,15)$. The main side effects of anthracyclines are cardiac toxicities including arrhythmia, heart failure, and myocardial damage (16); in addition to hair loss, anthracyclines can also decrease the amount of white blood cells and platelets, leading to anemia. Our current study was designed to investigate the effects of anthracyclines on glucose and lipid metabolism in breast cancer patients; the analysis showed that anthracycline-based chemotherapy was a protective factor for increased fasting blood glucose after chemotherapy in invasive cancer breast patients $(\mathrm{P}=0.022$, OR $=0.227,95 \%$ CI: $0.064-0.808)$. The fasting 
Table 1 General data of the two groups

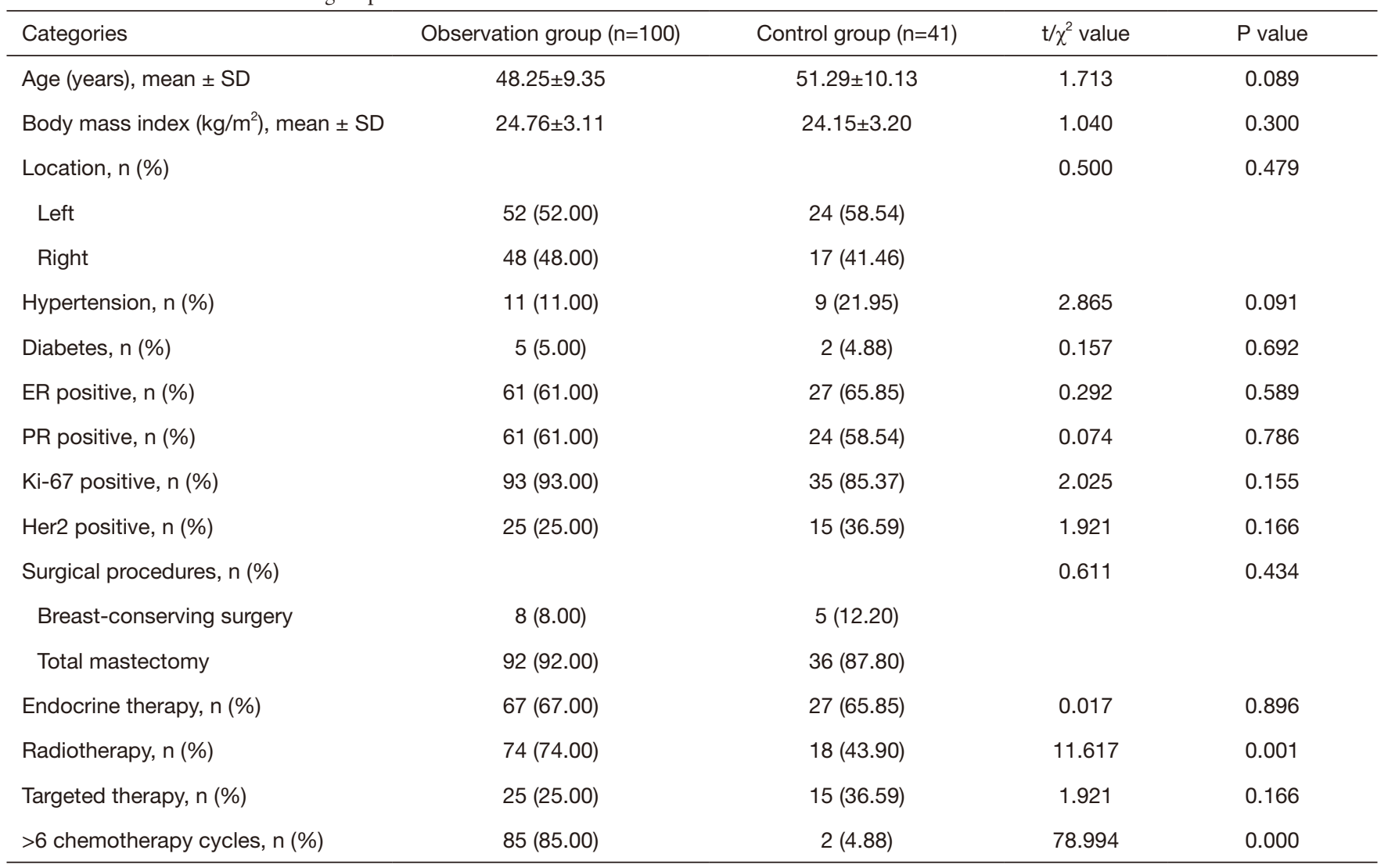

blood glucose was not significantly different between the observation group and control group before chemotherapy ( $\mathrm{P}>0.05)$. After chemotherapy, the fasting blood glucose significantly increased in the observation group $(5.31 \pm 0.98$ vs. $4.96 \pm 0.53, \mathrm{P}=0.031$ ).

As the postoperative recurrence and mortality rates gradually drop in breast cancer patients, the concern has increasingly shifted to the quality of life after breast cancer surgery. A recent study investigated the effects of postoperative chemotherapy on blood glucose and lipids in breast cancer patients (17). Anthracycline-based chemotherapy regimens are important postoperative chemotherapy strategies for breast cancer. The basic structure of an anthracycline is the anthracycline rings linked to an amino sugar by a glycosidic bond. Based on their chemical structures, anthracyclines are classified as antitumor antibiotics, which are chemical substances with antitumor activity produced by microorganisms. Mainly by embedding between DNA bases, they exert their antitumor cell effects by interfering with DNA transcription/messenger RNA synthesis, inhibiting DNA synthesis, generating oxygen radicals, and disrupting cellular membrane. A study performed in 2009 showed that neoadjuvant chemotherapy could lead to increased blood glucose in patients (18). In our current study, fasting blood glucose levels were significantly elevated in both groups after chemotherapy, which was consistent with the previous study. We also found that fasting blood glucose was not significantly different between the observation group and control group before chemotherapy $(\mathrm{P}>0.05)$. After chemotherapy, fasting blood glucose significantly increased in the observation group $(5.31 \pm 0.98 v$ vs. $4.96 \pm 0.53$, $\mathrm{P}=0.031)$. Further analysis showed that anthracycline-based chemotherapy was a protective factor for increased fasting blood glucose after chemotherapy in invasive cancer breast patients $(\mathrm{P}=0.022$, OR $=0.227,95 \%$ CI: 0.064-0.808), whereas receiving $>6$ cycles of chemotherapy was a risk factor for increased fasting blood glucose $(\mathrm{P}=0.014$, OR $=4.216,95 \%$ CI: 1.337-13.296). Therefore, anthracyclinebased chemotherapy regimens have less glycemic effects than non-anthracycline-based chemotherapeutic agents at lower cumulative doses, but their glycemic effects gradually 
Table 2 Comparison of glucose and lipid profiles before and after chemotherapy between the two groups

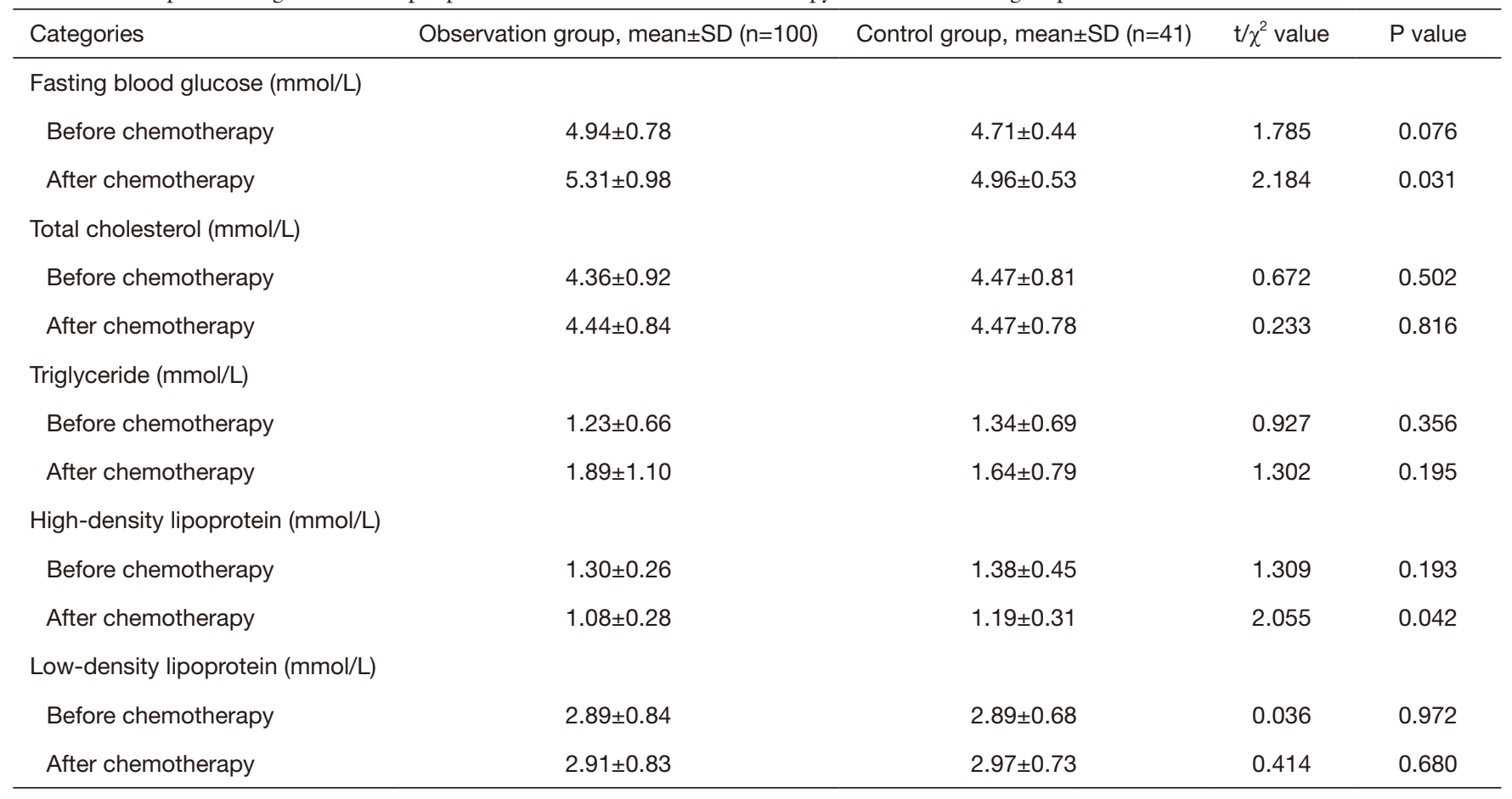

Table 3 Risk factors for increased fasting glucose after chemotherapy in patients with invasive breast cancer

\begin{tabular}{lccccc}
\hline Factors & B value & Standard error & Wald value & P value & 95\% confidence interval \\
\hline Constant coefficient & 0.520 & 0.601 & 0.747 & 0.387 & \\
$>6$ chemotherapy cycles & 1.482 & 0.648 & 6.029 & 0.014 & $4.216(1.337-13.296)$ \\
Radiotherapy & 0.291 & 0.423 & 0.474 & 0.491 & $1.338(0.584-3.068)$ \\
Anthracycline-based chemotherapy regimen & 1.482 & 0.648 & 5.237 & 0.022 & $0.227(0.064-0.808)$ \\
\hline
\end{tabular}

Table 4 Risk factors for reduction of high-density lipoprotein in invasive breast cancer patients after chemotherapy

\begin{tabular}{lccccc}
\hline Factors & B value & Standard error & Wald value & P value & 95\% confidence interval \\
\hline Constant coefficient & 1.265 & 0.433 & 8.528 & 0.003 & \\
$>6$ chemotherapy cycles & 0.176 & 0.699 & 0.063 & 0.801 & $1.192(0.303-4.693)$ \\
Radiotherapy & 0.394 & 0.464 & 0.721 & 0.396 & $0.675(0.272-1.674)$ \\
Anthracycline-based chemotherapy regimen & 0.051 & 0.745 & 0.005 & 0.945 & $0.950(0.221-4.093)$ \\
\hline
\end{tabular}

increase with increasing doses of anthracyclines.

Furthermore, our analysis showed a significant increase in triglyceride levels and a decrease in HDL in both groups after chemotherapy. It has been found that chemotherapy might also cause abnormalities in lipid metabolism in breast cancer patients, especially in younger patients (19). There were similar findings in our current study. However, in our current study, compared with non-anthracycline-based chemotherapy regimens, anthracycline-based chemotherapy regimens had no significant impact on blood lipid metabolism.

However, our study was with its shortages. First of all, 
this was retrospective study, so we failed to study other related factors in this study. For example, insulin resistance may be a risk factor for breast cancer, possibly through increased levels of oestrogens or insulin-like growth factor I. Insulin resistance has been associated with obesity, hypertension, dyslipidaemia and impaired glucose tolerance. But in this study, we failed to study these factors. Moreover, we failed to study the change of the body mass index in this study.

In summary, anthracyclines have little effect on fasting blood glucose in breast cancer patients; however, the incidence of abnormal blood glucose metabolism gradually increases after prolonged anthracycline use. Compared with other chemotherapy drugs, anthracycline-based chemotherapy has no significant impact on blood lipid metabolism.

\section{Acknowledgments}

Funding: Top notch youth training program of Military Medical Science (20QNPY113).

\section{Footnote}

Reporting Checklist: The authors have completed the STROBE reporting checklist. Available at http://dx.doi. org/10.21037/apm-21-533

Data Sharing Statement: Available at http://dx.doi. org/10.21037/apm-21-533

Conflicts of Interest: All authors have completed the ICMJE uniform disclosure form (available at http://dx.doi. org/10.21037/apm-21-533). The authors have no conflicts of interest to declare.

Ethical Statement: The authors are accountable for all aspects of the work in ensuring that questions related to the accuracy or integrity of any part of the work are appropriately investigated and resolved. All procedures performed in this study involving human participants were in accordance with the Declaration of Helsinki (as revised in 2013). This study was approved by the ethics committee of the First Medical Center of the Chinese People's Liberation Army General Hospital (2021 166) and individual consent for this retrospective analysis was waived.

Open Access Statement: This is an Open Access article distributed in accordance with the Creative Commons Attribution-NonCommercial-NoDerivs 4.0 International License (CC BY-NC-ND 4.0), which permits the noncommercial replication and distribution of the article with the strict proviso that no changes or edits are made and the original work is properly cited (including links to both the formal publication through the relevant DOI and the license). See: https://creativecommons.org/licenses/by-nc-nd/4.0/.

\section{References}

1. Oliver J, Quezada Urban R, Franco Cortés CA, et al. Latin American Study of Hereditary Breast and Ovarian Cancer LACAM: A Genomic Epidemiology Approach. Front Oncol 2019;9:1429.

2. Prysyazhnyuk AY, Bsmall C, Romanenko A, et al. Epidemiology of Breast Cancer in Ukraine with Consideration of the Factors of the Chornobyl Accident. Probl Radiac Med Radiobiol 2019;24:150-68.

3. Adejumo AA, Ajamu O, Akanbi O, et al. Epidemiology and Challenges of Managing Breast Cancer in Keffi, NorthCentral Nigeria: A Preliminary Report. Niger Med J 2019;60:193-97.

4. Zhang Z, Rao R, Mango V, et al. Presentation of selfdetected breast mass in minority women with limited access to care: Can self-examination assist in early cancer detection? Clin Imaging 2021;70:89-92.

5. Zejda JE, Kaleta A. Modes of Early Detection of Breast Cancer in Katowice Region, Poland. Int J Environ Res Public Health 2020;17:3390.

6. Yuniar P, Robinson S, Moorin R, et al. Economic Evaluation of Breast Cancer Early Detection Strategies in Asia: A Systematic Review. Value Health Reg Issues 2020;21:252-63.

7. Yoshinami T, Kagara N, Motooka D, et al. Detection of ctDNA with Personalized Molecular Barcode NGS and Its Clinical Significance in Patients with Early Breast Cancer. Transl Oncol 2020;13:100787.

8. Sutton TL, Johnson N, Schlitt A, et al. Surgical timing following neoadjuvant chemotherapy for breast cancer affects postoperative complication rates. Am J Surg 2020;219:741-45.

9. Srour MK, Lee M, Walcott-Sapp S, et al. Thirtyday postoperative morbidity in patients with breast cancer following neoadjuvant chemotherapy. Am J Surg 2020;220:660-64.

10. Malekzadeh P, Cowan K, Steinberg SM, et al. Twentyfive-Year Follow-up of a Prospective Randomized Trial 
Comparing Preoperative Versus Postoperative FLAC/

Granulocyte Colony-Stimulating Factor Chemotherapy for Stage II Breast Cancer. Am J Clin Oncol 2020;43:334-39.

11. Buch K, Gunmalm V, Andersson M, et al. Effect of chemotherapy and aromatase inhibitors in the adjuvant treatment of breast cancer on glucose and insulin metabolism-A systematic review. Cancer Med 2019;8:238-45.

12. Kaboré EG, Guenancia C, Vaz-Luis I, et al. Association of body mass index and cardiotoxicity related to anthracyclines and trastuzumab in early breast cancer: French CANTO cohort study. PLoS Med 2019;16:e1002989.

13. Coutinho Cruz M, Cruz L, Branco M, et al. Threedimensional speckle-tracking echocardiography for the global and regional assessments of left ventricle myocardial deformation in breast cancer patients treated with anthracyclines. Clin Res Cardiol 2020;109:673-84.

14. Abdel-Rahman O. Outcomes of early-stage breast cancer patients treated with sequential anthracyclines-taxanes in relationship to relative dosing intensity: a secondary analysis of a randomized controlled trial. Clin Transl Oncol 2019;21:239-45.

Cite this article as: Qi A, Li Y, Yan S, Sun H, Chen Y. Effect of anthracycline-based postoperative chemotherapy on blood glucose and lipid profiles in patients with invasive breast cancer. Ann Palliat Med 2021;10(5):5502-5508. doi: 10.21037/apm-21533
15. Ma F, Ouyang Q, Li W, et al. Pyrotinib or Lapatinib Combined With Capecitabine in HER2-Positive Metastatic Breast Cancer With Prior Taxanes, Anthracyclines, and/or Trastuzumab: A Randomized, Phase II Study. J Clin Oncol 2019;37:2610-19.

16. Kimmick G, Dent S, Klem I. Risk of Cardiomyopathy in Breast Cancer: How Can We Attenuate the Risk of Heart Failure from Anthracyclines and Anti-HER2 Therapies? Curr Treat Options Cardiovasc Med 2019;21:30.

17. Thakur SB, Horvat J, Hancu I, et al. Quantitative in vivo proton MR spectroscopic assessment of lipid metabolism: Value for breast cancer diagnosis and prognosis. J Magn Reson Imaging 2019;50:239-49.

18. Hickish T, Astras G, Thomas P, et al. Glucose intolerance during adjuvant chemotherapy for breast cancer. J Natl Cancer Inst 2009;101:537.

19. Tian W, Yao Y, Fan G, et al. Changes in lipid profiles during and after (neo)adjuvant chemotherapy in women with early-stage breast cancer: A retrospective study. PLoS One 2019;14:e0221866.

(English Language Editor: J. Gray) 